\title{
Comparative Aspects in Interictal, Intraictal and Postictal Electroencephalogram in Dogs with Idiopathic Epilepsy
}

\author{
Gabriela-Dumitriţa STANCIU, Mihai MUSTEAŢĂ, Mihaela ARMAȘU, Gheorghe SOLCAN* \\ Faculty of Veterinary Medicine, Department of Internal Medicine / Neurology \\ University of Agricultural Sciences and Veterinary Medicine "Ion Ionescu de la Brad" Iasi Romania, 3-8 M. \\ Sadoveanu Alley, 700489 \\ *Corresponding author: gsolcan@uaiasi.ro
}

Bulletin UASVM Veterinary Medicine 72(2) / 2015,

Print ISSN 1843-5270; Electronic ISSN 1843-5378

DOI:10.15835/buasvmcn-vm: 11358

\begin{abstract}
Epilepsy is a chronic brain disease, of varied etiology, defined by the presence of the seizures of definite epileptic nature and by evolutional criteria, made of their tendencies to repeat in absence of triggering factors, known at variable intervals. The diagnosis of this disease is based on the clinical features and electrophysiology of the brain. Electroencephalogram (EEG) is the efficiently electrobiological test assessing the impact of epilepsy on activity of the brain.

The aims of this study are to describe the interictal, intraictal and postictal parameters, and to evaluate the clinical usefulness of the EEG recording in dogs with idiopathic epilepsy, using induced sleep as activation method.

EEG was performed on 27 dogs with idiopathic epilepsy. Electrical potentials acquisition was performed using the electroencephalograph Neurofax S, MEB 9400K Nihon Kohden. Before the test, all dogs were sedated with medetomidine hydrochloride $30 \mu \mathrm{g} / \mathrm{kg}$ inj. i.m. Stainless steel needle electrodes were subcutaneously placed, in an 8 channel bipolar montage, according to the model of Redding and Knecht (1984).

The visual and quantitative analysis of the electroencephalographic tracks in idiopathic epilepsy revealed a background activity with a high instability and diversity of aspects, as there was more discordance between the electrical and clinical findings of the epilepsy. During interictal period, in incipient cases and onset of epilepsy, the EEG alterations were discrete, resuming to a couple of overvaulted peaks and ample lent theta waves on a normal background track. When epilepsy had a longer evolution, the background activity showed an intersection of slow waves with abnormally frequent waves, rich in epilpeptiform interictal discharges like: fast spike, slow waves, polyspike and typical or atypical spike-wave complexes. The intraictal period was characterized by electrical crisis, suddenly appeared on all derivations, then intensified by neuronal recruiting phenomenon and in 2-3 seconds the EEG anomalies spread in all brain areas, as epilpeptiform discharges became bilateral synchronous. Postictal EEG was characterized by a much flattened aspect of the tracks, almost isoelectric.

In conclusion, EEG gives valuable information about parameters and the severity of changes induced by epilepsy. EEG recorded using as an induced sleep activation method is the main way which proves the presence of an epileptic focus in the absence of clinical sings.
\end{abstract}

Keywords: dog, electroencephalogram, idiopathic epilepsy.

\section{INTRODUCTION}

In veterinary neurology, the terms used to define a disease are often borrowed from the human literature but are not used consistently. Definitions for epilepsy in the veterinary literature include recurrent seizures (Knowles, 1998), a chronic recurrence of seizures without a demonstrable substrate (Jaggy and Heynold,
1996) and 2 or more recurrent seizures (Berendt, 2005). In dogs, idiopathic epilepsy (IE) is defined as recurrent seizures for which no underlying abnormality can be identified (Knowles, 1998).

Naturally occurring epilepsy is reported in many animal species including rodents, dogs, cats, horses, cattle, goats, and non-human primates (Chandler, 2006). Seizures are also the most 
frequent neurological problem in dogs, with an estimated prevalence from 0.5 to $5.7 \%$ (Bielfelt et al., 1971; Schwartz-Porsche, 1994; Knowles, 1998; Licht et al., 2002; O’Brien, 2003; Patterson et al., 2005; Chandler, 2006) in dogs and approximately $0.5 \%$ in cats (Schwartz-Porsche, 1994); comprising $2-3 \%$ of canine patients treated at veterinary teaching hospitals (Podell et al., 1995) and involving $10 \%$ of neurological problems (Jaggy and Bernardini, 1998). Seizures are also estimated to be responsible for $8 \%$ of dog deaths in the UK (Armașu et al., 2014).

Diagnosis of epilepsy is fundamentally based on the history of recurrent seizures, but general physical and detailed neurological examinations are basic assessments that should be performed on every animal with the history of seizures. Electroencephalogram (EEG) is the efficiently electrobiological test to diagnose and manage epilepsy; trying to assess the impact of seizures on electrical activity of the brain. The EEG is a noninvasive electrodiagnostic investigation, which can be faithfully repeated without limits, with no adverse effects (Aminoff, 2005) to monitor and evaluate functional activity of the epileptic brain in real time.

The aims of this study are to describe the interictal, intraictal and postictal parameters, and to evaluate the clinical usefulness of the EEG recording in dogs with $\mathrm{IE}$, using as activation method induced sleep.

\section{MATERIALS AND METHODS}

27 dogs with IE were selected for the study. The study was conducted at the Faculty of Veterinary Medicine, Internal Medicine (Neurology), and was performed in accordance with the guidelines and upon approval of the Animal Care Committee of the University of Agricultural Sciences and Veterinary Medicine, Iasi, Romania. A complete history, clinical and neurological examinations and laboratory diagnostics, including complete blood count, serum chemistry profile, and urine analysis were carried out in order to rule out extracranial causes for seizures.

Serum chemistry profile consisted of glucose, total protein, albumin, globulin, blood urea nitrogen, creatinine, total bilirubin, alanine aminotransferase, aspartate aminotransferase, alkaline phosphatase, creatine kinase, cholesterol, and electrolytes $(\mathrm{Na}, \mathrm{K}, \mathrm{Ca}, \mathrm{P}, \mathrm{Mg})$.
To exclude the systemic conditions (liver and kidney diseases or metastatic neoplasia), in all patients a radiographic evaluation (tympanic bulla and thoracic cavity) and abdominal ultrasonography examination were performed.

The epileptic patients in our study belonged to the following breeds: German Shepherd ( $n=5$, Bichon $(\mathrm{n}=7)$, Tosa Innu $(\mathrm{n}=1)$, common breed $(n=3)$, Yorkshire Terrier $(n=3)$, Cocker $(n=2)$, Labrador $(n=4)$ and Dalmatian $(n=2)$, both sexes, between 2-6 years old.

The IE diagnosis was established when the results of hematological and biochemical analyses were in physiological limits, and subsequent to computer tomography/magnetic resonance imaging and LCR examinations, no changes were detected. The epilepsy manifested by generalized form. An epileptic attack was considered generalized, when the motor activity included the whole body.

The electroencephalogram was performed under sedation, using medetomidine (Domitor, Pfizer) in a dosage of $30 \mu \mathrm{g} / \mathrm{kg}$ administered intramuscularly, in order to eliminate the artifacts triggered by the muscular contractions. The acquisition of the biopotentials was made with Neurofax electroencephalograph (Nihon Kohden) for 30 minutes. The electrodes were subcutaneously placed over the right/left frontal and right/left occipital lobes and vertex and an 8 channel bipolar montage was used according to the method of Redding and Knecht (1984) (Fig.1.). Electrodes' nomenclature is similar to the one described by 10-20 system in human medicine (Aminoff, 2005; Nordli et al., 2011).

The parameters used for each electroencephalographic recording were: sensitivity: $70 \mu \mathrm{V}$, time constant: 0.3 seconds, filter pass - down of $70 \mathrm{~Hz}$, filter pass - up $30 \mathrm{~Hz}$ and electrode impedance $<10 \Omega$.

During the EEG recording we looked for possible epileptiform discharge, especially interictal epileptiform discharge (DIEs) representing the basic elements of EEG diagnosis in epilepsy (Niedermeyer, 2005). EEG visual analysis also, was based of the frequency and amplitude of background rhythms interpretation, the presence or absence of transient events, the presence and distribution of abnormal events and the precise characteristics of abnormal events. 
The EEG records were visually examined in bipolar montage. The sleep stage, possible normal variants, or epileptiform findings, without knowing the clinical status of the dogs were described. For all dogs, 30 replications of 2 -second unsuppressed epochs without epileptic activity were analyzed. Background activity was calculated and averaged for each channel, with the same acquisition way and with an integrated software program Fast Fourier Transform. The spectral bands of delta $(0.5-4 \mathrm{~Hz})$, theta (4- 8 $\mathrm{Hz})$, alpha $(8-13 \mathrm{~Hz})$ and beta $(13-30 \mathrm{~Hz})$ were calculated and expressed as relative power (\%). In order to minimize errors through different skull sizes, forms and thicknesses, the relative power of the spectral bands was calculated for every lead.

\section{RESULTS AND DISCUSSION}

At the visual examination of the EEG recordings, all dogs exhibited a high-voltage lowfrequency background activity, probably as a consequence of using medetomidine, corresponding to the study developed by Short et al. (1992), which describe the inhibitor effect of the anesthetics from agonist alfa-2-adrenoceptor group upon increased frequencies.

In interictal period, the visual analysis of the electroencephalographic tracks showed a physiological background activity in 5/27 patients $(18.51 \%)$ with IE. In human medicine,

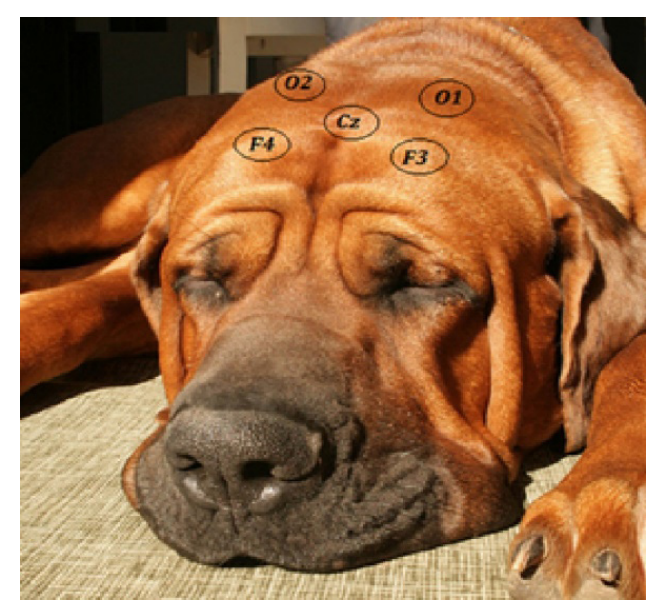

Fig. 1. The electrodes were positioned according to the model of Redding and Knecht (1984). F4 is the frontal right, F3 frontal left; $\mathrm{Cz}$ represents vertex, O1 left and $\mathrm{O} 2$ right occipital electrodes. The reference electrode was on the ridge of the nose between the eyes. The following leads were used throughout the manuscript: F3-F4, F4-

O2, O1-O2, O1-F3, F3-Cz, Cz-F4, Cz-O1, Cz-O2 approximately $20 \%$ of the patients with clinically verified epileptic seizures do not show any electrogenesis disorder, the anomalies appearing only in the moments the out breaks set off (Dumitru, 2002). Intercritical EEG tracts remained completely the same in the case of epilepsy with rare seizures only. In contrast with these were those where background EEG was disrupted $15 / 27$ cases ( $55.55 \%$ ), from the amplitude point of view or only of the basic frequency rhythms rich in DIEs represented by fast spike (Fig. 2), slow waves (Fig. 3), poly-spikes and typical or atypical spike-wave complexes (Fig. 4). There were also EEG tracks slightly modified, 6/27 (22.22 \%), by decrease or increase of the brain rhythms, permanently associated to the important increase of the amplitude. In severe cases of epilepsy $(n=1$; $3.70 \%)$, EEG showed an important changed and uneven bioelectrical activity, imitating the described classical aspect under the name of hypsarrythmia (Nordli et al., 2011) - Fig. 5.

In a study conducted by Pillai and Sperling (2006), DIEs have been detected by EEG in $29 \%$ to $55 \%$ of epileptic human's patients. When outpatient EEG was repeated up to four times, detection of DIEs could rise up to $90 \%$. EEG sensitivity is highest in children and lowest in elderly patients (Stern, 2005). Importantly, not all interictal spikes and sharp waves are associated with epilepsy (i.e. benign epileptiform transients of sleep, wicket spikes, rhythmic midtemporal

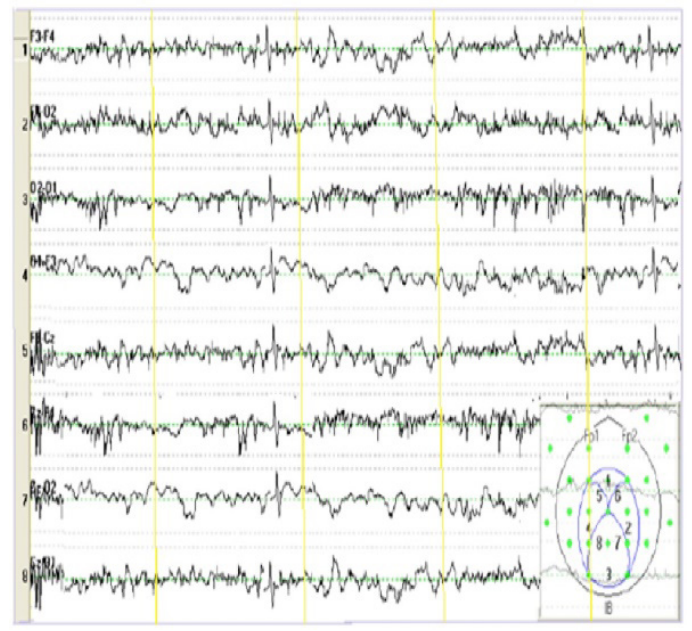

Fig. 2. EEG of a Labrador dog, 3 years old with IE. Beta dominant background activity with fast spikes $(60 \mu \mathrm{V})$ recorded in all derivations 
theta discharges, vertex sharp waves, and midline theta rhythm) and are considered as benign findings unrelated to seizure disorders (Pillai and Sperling, 2006).

The electrical crisis or intraictal period was recorded in only 4 patients in the study and suddenly appeared on all derivations, then intensified by neuronal recruiting phenomenon and in 2-3 seconds the EEG anomalies spread in all brain areas, as DIEs became bilateral synchronous. This aspect corresponds to the moment in which the activity of the entire brain

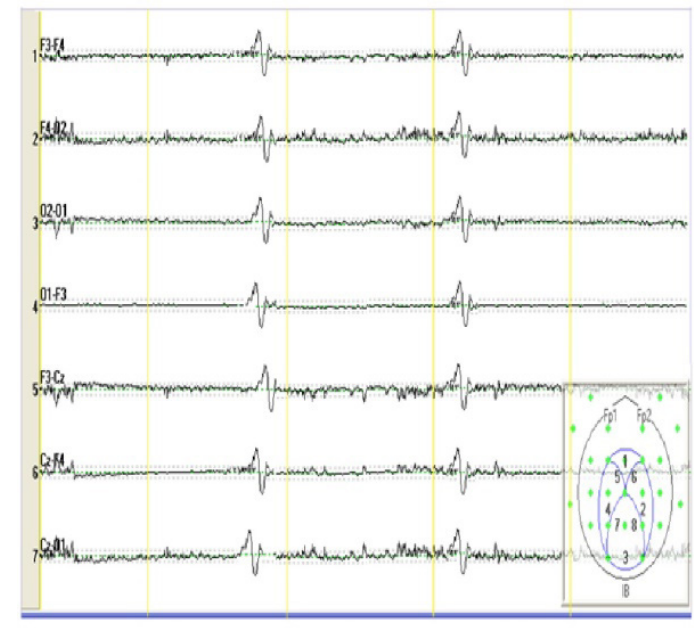

Fig. 3. Hyper-synchronous slow waves on a background activity represented by beta rhythm with a frequency of $20-25 \mathrm{~Hz}$ and amplitude of $30 \mu \mathrm{V}$ recorded in a Dalmatian dog, 2.5 years old with EI

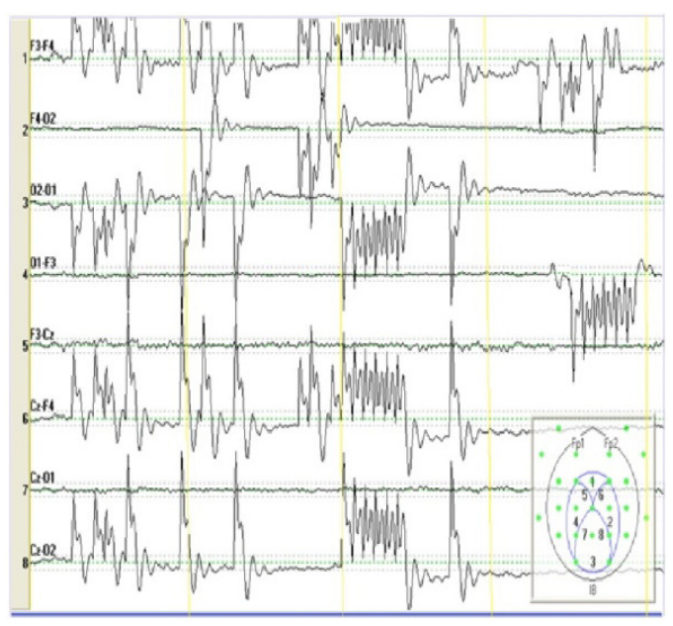

Fig. 4. Poly-spike and atypical spike-wave complexes on a hypovaulted background activity recorded in a cross breed dog, 4-year-old adapted to the rhythm developed by the epileptic site (Short et al., 1992; Dumitru, 2002). The access was characterized by a succession of peaks with a frequency of 15-35 cycles/second, increasing progressively in amplitude, reaching values of up to $250-500 \mu \mathrm{V}$ (Fig. 6).

After these ample and rapid anomalies, which correspond to the tonic phase of the seizure, the morphology of the electrical paroxysms changed. In a couple of seconds, the rhythm of epileptic

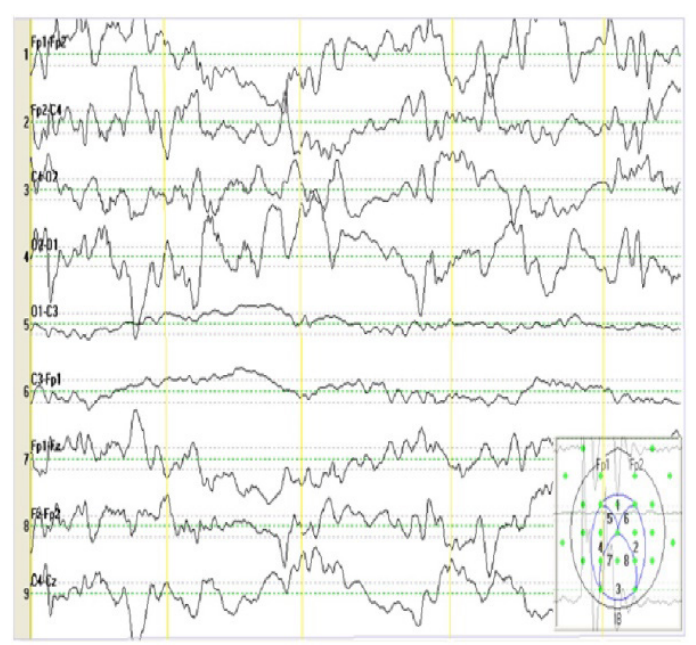

Fig. 5. The background activity amplitude is approximately $300 \mu \mathrm{V}$ and includes a mixture of frequencies without normal consistent rhythms. Multiple spikes are present and have a bilateral posterior predominance. The EEG was recorded from a 1.4-yearold Tosa Innu dog, in bipolar montage

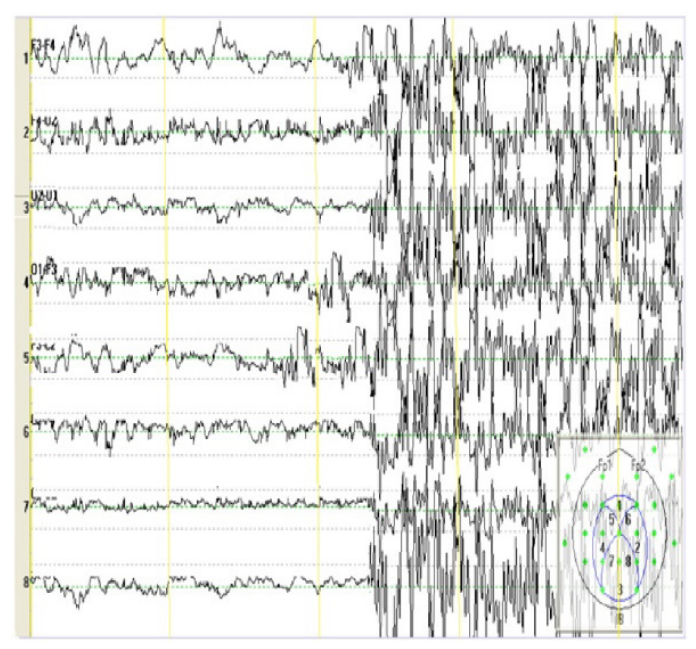

Fig. 6. EEG during an epileptic seizure recorded in a 4 years old German Sheppard dog 
discharges decreased to 3-5 cycles/second, then setting up progressively during postictal period.

Postictal EEG was characterized by a much flattened aspect of the tracks. After this apparently electrical silence, sometimes, for intervals of 10-15 seconds, short bursts of peaks (Fig.7) or degraded spike-wave complexes appeared, clinically followed each time by generalized twinges or synchronous myoclonic bursts in the extremities.

In our study, the results of the quantitative EEG showed a higher prevalence of slow rhythms (delta and theta) in all dogs, while fast rhythms (alpha and beta) were poorly represented. This seems to be a common EEG background pattern in sedated dogs (Srenk and Jaggy 1996, Itamato et al. ,2001, Bergamasco et al. ,2003, Pellegrino and Sica, 2004). An increase in the beta band was observed in a previous EEG study of healthy human volunteers after the oral administration of phenobarbital, and in a study of epileptic patients, although the results failed to reach statistical significance (Sannita et al., 1980, Herkes et al., 1993). A dose-dependent increase in beta and theta bands after phenobarbital administration has been reported in rats as well (Sato, 1980). Theta rhythm is associated with the use of sedatives, such as barbiturates or neuroleptics, in humans (Stern, 2005). Phenobarbital seems to increase the theta, alpha, and beta bands, and to decrease the delta one. Our results indicate that treatment

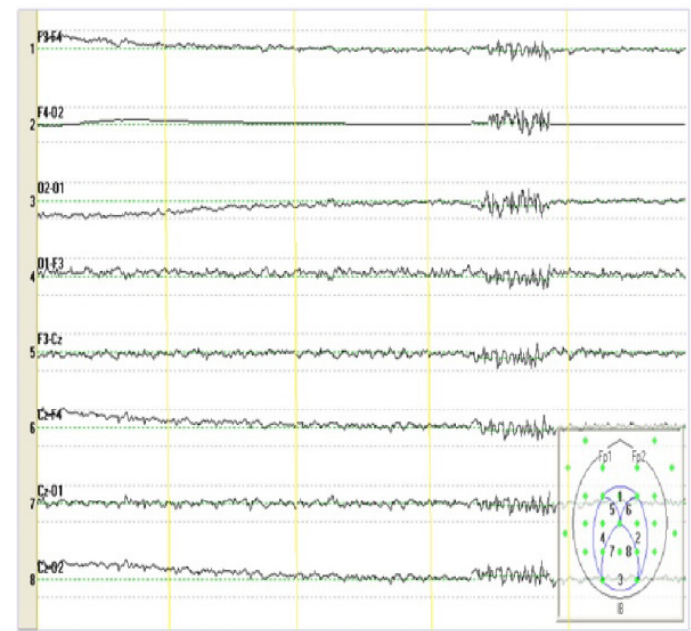

Fig. 7. Short bursts of spikes recorded during postictal period. 3 years old Labrador male dog with antiepileptic drugs should be considered when interpreting the data from quantitative EEG. However, in epileptic patients whose visual evaluation of interictal EEG remains normal, information from quantitative analysis of EEG might be complementary.

\section{CONCLUSION}

EEG gives valuable information about parameters and the severity of changes induced by epilepsy and is the main way to prove the presence of an epileptic focus in the absence of clinical signs. Concomitant use of visual and quantitative analysis of EEG in epileptic patients is advised as complementary information can be achieved.

Acknowledgment - this paper is published under the frame of European Social Fund, Human Resources Development Operational Program 2007-2013, project no. POSDRU/159/1.5/S/ 132765.

\section{REFERENCES}

1. Aminoff MJ (2005). Electroencephalography: General Principles and Clinical Applications. In: Aminoff, MJ (Ed). Electrodiagnosis in Clinical Neurology, Elsevier, Philadelphia, 37-84.

2. Armaşu M, Packer RMA, Cook S, Solcan G, Volk HA (2014). An exploratory study using a statistical approach as a platform for clinical reasoning in canine epilepsy. The Veterinary Journal 202:292-296.

3. Berendt M (2005). Epilepsy. In: Vite CH, Braund KG (eds). Braund's Clinical Neurology in Small Animals. International Veterinary Information Service, Ithaca, NY, USA, 35-47.

4. Bergamasco L, Accatino A, Priano L, Neiger-Aeschbacher G, Cizinauskas S, Jaggy A, (2003). Quantitative electroencephalographic findings in beagles anesthetized with propofol. Vet J 166:58-66.

5. Bielfelt SW, Redman HC, McClellan RO, (1971). Sire- and sex related differences in rates of epileptiform seizures in a purebred beagle dog colony. American Journal of Veterinary Research 32:2039-2048.

6. Chandler K (2006). Canine epilepsy: what can we learn from human seizure disorders? The Veterinary Journal 172:207-217.

7. Dumitru D (2002). Electrodiagnostic medicine. Hanley \& Belfus, Philadelphia, 29-64.

8. Herkes GK, Lagerlund TD, Sharbrough FW, Eadie MJ (1993). Effects of antiepileptic drug treatment on the background frequency of EEGs in epileptic patients. J Clin Neurophysiol 10:210-216. 
9. Itamoto K, Taura Y, Wada N (2001). Effect of medetomidine on EEG and use of a q-EEG for evaluating sedation levels in dogs. J Vet Med A 48:525-535.

10. Jaggy A, Bernardini M (1998). Idiopathic Epilepsy in 125 dogs: a long term study. Clinical and electroencephalographic findings. Journal of Small Animal Practice 39:23-29.

11. Jaggy A, Heynold Y (1996). Idiopathic epilepsy in the dog. Schweiz Arch Tierheilkd 138: 523-531.

12. Knowles K (1998). Idiopathic epilepsy. Clinical Techniques in Small Animal Practice 13: 144-151.

13. Licht MH, Harper KM, Lin S, Curtin JJ, Hyson LL, Willard K (2002). Clinical presentations of naturally occurring canine seizures: similarities to human seizures. Epilepsy and Behaviour 3:460-470.

14. Niedermeyer E, Schomer DL(2005). Historical aspects of EEG. In: Schomer DL, Lopez da Silva FH (eds). Niedermeyer`s electroencephalography. Basic principles, clinical applications, and related fields. 6th ed. Wolters Kluwer, Lippincott Williams \& Wilkins, 1-16.

15. Nordli D, Riviello J, Niedermeyer E (2011). Seizures and epilepsy in infants to adolescents. In: Schomer DL, Da Silva FL (eds).Niedermeyer's Electroencephalography. Basic principles, clinical applications, and related fields, Lippincott, Philadelphia, 479-540.

16. O'Brien D (2003). Pathogenesis of idiopathic epilepsy. In: Proceedings of the American College of Veterinary Internal Medicine Annual Forum, 404-406.

17. Patterson EE, Armstrong PJ, OBrien DP, Roberts MC, Johnson GS, Mickelson JR (2005). Clinical description and mode of inheritance of idiopathic epilepsy. In : English Springer Spaniels. Journal of the American Veterinary Medical Association 226:54-58.
18. Pellegrino FC, Sica REP (2004). Canine EEG recording technique: findings in normal and epileptic dogs. Clin Neurophysiol 115:477-487.

19. Pillai J, Sperling MR (2006). Interictal EEG and the diagnosis of epilepsy. Epilepsia 47:14-22.

20. Podell M (1995). The use of diazepam per rectum at home for the acute management of cluster seizures in dogs. Journal of Veterinary Internal Medicine 9:68-74.

21. Redding RW, Kneckt EC (1984). Atlas of electroencephalography in the dog and cat. Praeger Publisher, New York, USA.

22. Sannita WG, Rapallino MW, Rodriguez G, Rosadini G (1980). Effects and plasma concentrations of phenobarbital in volunteers. Neuropharmacology 19:927-930.

23. Sato H (1980). Relationship between serum levels and fast EEG activities in rats by a single administration of phenobarbital. Electroencephalogr Clin Neurophysiol 50:509-514.

24. Schwartz-Porsche D (1994). Seizures. In: Braund KG (ed). Clinical Syndromes in Veterinary Neurology, Mosby, St Louis, 234-251.

25. Short CE, Raiha JE, Raiha MA, Otto K (1992). Comparison of neurologic responses to the use of medetomidine as sole agent or preanesthetic in laboratory beagles. Acta Veterinaria Scandinavica 33:77-88.

26. Srenk P, Jaggy A (1996). Interictal EEG findings in a Golden Retrievers with idiopatic epilepsy. J Small Anim Pract 37:317-321.

27. Stern JM (2005). Interictal epileptiform discharges. In: Stern JM, Engel J (eds). Atlas of EEG patterns, Lippincott Williams \& Wilkins, 161-176. 